\title{
The relationship between interference control and sense of presence in virtual environments
}

\author{
Boris B. Velichkovsky \\ Faculty of Psychology, Lomonosov Moscow State University, Moscow, Russia \\ Corresponding author. E-mail: velitchk@mail.ru
}

Background. The sense of presence is an important aspect of interaction with virtual reality applications. Earlier we suggested that presence can depend on cognitive control. The latter is a set of meta-cognitive processes which are responsible for configuring the cognitive system for the accomplishment of specific tasks with respect to a given context. In particular, cognitive control helps in preventing interference from the task-irrelevant variables.

Objective. This study aimed at investigation of the possible relationship between interference control and aspects of presence.

Design. Thirty-nine subjects ( 32 female and 7 male, aged 18 to 27 years) participated in the study. The subjects were assessed via a battery of interference control tasks (Flanker Task, Go/No Go task, antisaccade task) and performed a virtual scenario (navigating within an array of randomly placed virtual digits in correct numerical order) in high-immersion (CAVE) and low-immersion (standard computer display) virtual environments. Afterwards, the subjects completed a Russian version of the ITC-Sense of Presence inventory.

Results. We found that interference control is generally related to the sense of presence, especially in the CAVE (high-immersion) environment. Sensory interference control was most strongly associated with various aspects of presence (overall presence score, spatial presence, and emotional involvement). Motor interference control was associated with spatial presence and emotional involvement, but this relationship was weaker than was the case with sensory interference control. Low-immersion virtual environments attenuate some of these links between interference control and presence so that only sensory interference control remains a notable predictor of presence. Conclusion. Interference control is positively associated with presence in virtual environments with varying immersion levels. This may reflect a more general cause-and-effect relationship between cognitive control and the feeling of presence in virtual reality.

Keywords: virtual reality, presence, interference, cognitive control, attention, flanker task, antisaccade task, Go/No Go task 


\section{Introduction}

Virtual reality (VR) applications are being increasingly used in science and technology. An important aspect of interacting with VR is presence (Sanchez-Vives \& Slater, 2005; Diemer et al., 2015). Presence is associated with the feeling of being located in the virtual environment and the realness of interacting with virtual objects. It can be defined as the "perceptual illusion of non-mediation" (Lombard \& Ditton, 1997). This means that presence is experienced as if the virtual environment is real and there is no or little conscious awareness of either the real environment, or of the technology used to produce the virtual experience. It is obvious that presence is the very heart of the idea of VR; if there is no presence, the user experiences discomfort when interacting with a VR application, and there is little opportunity for virtual reality to become the user's reality. The feeling of presence exerts a strong influence on the quality of interaction with VR systems. It is easy to see that optimizing presence is an important objective for designers of VR applications.

There are many determinants of presence, both technological and psychological. Technological determinants of presence mostly concern the fidelity of the VR presentation. The rule of thumb is that the more realistic the output of the presentation system is the more presence emerges in the user. Thus, full immersion (via head-mounted displays or the CAVE system), high frame rate, high resolution, the use of spatial sound, low feedback latency, the possibility of interacting with virtual objects should produce increased presence. However, this is not always the case. An example is the so-called "book paradox": a well-written book produce high presence and involvement in the reader although there is no immersion at all. Presence is a subjective phenomenon and therefore psychological factors are at least as important as the visual fidelity of the presentation.

Research on the psychological determinants of presence has produced some interesting results. Presence seems to be dependent upon gender, age, and personality variables (Sacau et al., 2008). Among the latter, extraversion, locus of control, openness to experience, and psychological absorption were shown to be of relevance (Sas, 2004; Baños et al., 1999). However, the results have been mixed. For instance, extraversion was shown to be related to presence both positively and negatively. These mixed results indicate that personality traits may not be an appropriate level of organization on which to search for psychological determinants of presence. Previously, it has been suggested that presence can depend on cognitive control (Velichkovsky, 2014). Cognitive control is a set of meta-cognitive processes which are responsible for configuring the cognitive system for the accomplishment of specific tasks with respect to a given context (Notebaert \& Verguts, 2008). As such, cognitive control may be crucially responsible for re-configuring the cognitive system towards optimal interaction with a VR environment which is different from interaction with the real environment. In this study, we investigate whether presence is related to a fundamental aspect of cognitive control - specifically, control of interference.

Control of interference (or inhibition) is considered a basic function of cognitive control (Miyake et al., 2000). It comprises a set of related functions (Friedman \& Miyake, 2004). These include control of sensory interference (interference produced by the presence of irrelevant sensory stimuli); control of cognitive inter- 
ference (control of irrelevant representations activated in the course of cognitive processing and competing for processing resources, including proactive and reactive interference phenomena in memory); and control of inappropriate response tendencies. Inhibition of inappropriate saccadic responses (as measured by the antisaccade task) is a special class of inhibition functions, related but not identical to the inhibition of inappropriate motor responses. These functions are functions of voluntary interference control to be differentiated from automatic inhibition phenomena like inhibition-of-return. Research shows that inhibition functions are at the core of the more complex executive functions responsible for voluntary control of behavior (Friedman \& Miyake, 2004). The age-related decrease in the efficiency of interference control may be the cause of cognitive decline observed among older persons (Hasher \& Zacks, 1988).

There are several paths through which interference control may be related to presence. First, control of sensory interference may help the user devote attention to the virtual environment and ignore distracting stimuli from the now irrelevant real environment. This is the more important as the investment of attentional resources is considered to be an objective indicator of presence (Draper et al., 1998). Second, control of cognitive interference helps the mind free central resources from processing thoughts which are irrelevant to the virtual environment and concentrate on building an appropriate mental model of interacting with the virtual environment. Third, efficient control of eye movements would assist the user in directing his/her overt attention toward the virtual environment with the aim of preferentially processing VR stimuli. Fourth, control of motor responses would help the user to selectively attend to responses appropriate to the given virtual stimulation. In sum, interference control may assist the user in processing the stimuli, representations, and responses relevant to interacting with the virtual environment and thus help him/her ignore irrelevant stimuli, representations, and responses which would otherwise hinder the interaction with the virtual environment.

In the present study, correlations between the efficiency of interference control and the subjective sense of presence are investigated. Several interference control tasks are used to assess different aspects of interference control. The aim of the study is to show that there is a positive relationship between the efficiency of interference control and the sense of presence. To the best of our knowledge, this is the first study aimed at demonstrating this relationship. The results of the study will help to elucidate the cognitive mechanisms involved in the emergence of the sense of presence while interacting with virtual environments. They can also have practical implications, since finding stable relationships between cognitive control variables and presence may help in designing more effective VR applications, and in the selection of users for interacting with virtual environments.

\section{Method}

\section{Subjects}

Thirty-nine subjects aged 18 to 27 years -32 female and 7 male-all students at M.V. Lomonossov-Moscow State University, took part in the study. 


\section{Interference control tasks: Flanker Task}

The stimuli were five horizontally oriented arrows, arranged in a congruent $(>>>>>$, $<<<<<)$ or an incongruent $(>><>>, \quad<<><<)$ order. The subject's task was to identify the direction of the middle arrow by pressing a key. The subject was given a training series with 36 trials and a main series with four blocks. In each block, every sequence of arrows was presented 36 times. The presentation time was 1500 milliseconds (ms), with an interstimulus interval of $1000 \mathrm{~ms}$. Registered were the subject's reaction time $\left(\mathrm{T}_{\mathrm{av}}\right)$ and accuracy $\left(\mathrm{A}_{\mathrm{av}}\right)$ in general and for each trial type $\left(\mathrm{T}_{\text {con }}, \mathrm{A}_{\text {con }}\right.$, $\mathrm{T}_{\text {inc }}, \mathrm{A}_{\text {inc }}$ ), and time $\left(\mathrm{T}_{\text {int }}\right)$ and accuracy $\left(\mathrm{A}_{\text {int }}\right)$ related interference index (computed as the difference in responses between incongruent and congruent trials).

\section{Interference control tasks: Go/No Go task}

The stimuli were a target stimulus (X, 80\% of presentations) and distractor stimuli (A, Г, E, И, К, Л, M, H, П, Т, О, Э, Ю, Я). The stimuli were presented randomly in the center of the screen. The subject's task was to press a key if the target stimulus was presented. The presentation time was $300 \mathrm{~ms}$ with an interstimulus interval of $700 \mathrm{~ms}$. There was a training series with 20 trials and a main series with 200 trials. Registered were reaction time $\left(\mathrm{T}_{\mathrm{av}}\right)$, accuracy $\left(\mathrm{A}_{\mathrm{av}}\right)$, the number of hits $\left(\mathrm{N}_{\text {hit }}\right)$ and false alarms $\left(\mathrm{N}_{\mathrm{fa}}\right)$.

\section{Interference control tasks: Antisaccade task}

A fixation point was presented in the middle of the screen for a varying amount of time (1500 to $3500 \mathrm{~ms}$, in increments of $250 \mathrm{~ms}$ ). A visual distractor (a square with a side of $0.4^{\circ}$ ) was presented on the a randomly selected half of the screen for $200 \mathrm{~ms}$. The presentation of the distractor was followed by the presentation of the target stimulus (an arrow pointing left or right) in the opposite half of the screen for a very short period of $100 \mathrm{~ms}$; the stimulus was masked after presentation. The subject's task was to identify the orientation of the target stimulus by pressing a key: a procedure which implies suppressing the reflexive saccade towards the distractor. There was a training series of 16 trials and a main series of 96 trials. Registered were reaction times $\left(\mathrm{T}_{\mathrm{av}}\right)$ and accuracy $\left(\mathrm{A}_{\mathrm{av}}\right)$.

\section{Presence questionnaire}

The subjective feeling of presence was assessed with a Russian version of the ITCSense of Presence Inventory (Lessiter et al., 2001). This is a questionnaire of 44 items with subscales of Spatial Presence (SP), Naturalness (N), Emotional Involvement (EI), and Negative Effects (NE). Spatial Presence pertains to the illusion of being transferred to the virtual environment. Naturalness pertains to ease of understanding the virtual environment. Emotional Involvement pertains to the sense of engagement and its pleasantness while interacting with the virtual environment. Negative Effects pertains to vestibular disturbances which may emerge as a consequence of increased presence (nausea). An overall index of presence (Presence) was obtained by summing the scores for all items. 


\section{Virtual scenario}

To assess the sense of presence we needed a task which required orientation and movement in a virtual environment and active interaction with virtual objects. We created a virtual task which consisted in navigating within an array of randomly placed in a rectangular virtual space with a side of $20 \mathrm{~m}$ digits in correct numerical order. This virtual scenario was presented either in a high-immersion CAVE system or by the means of low-immersion standard 19" computer display. For each type of presentation, there was a training session (digits from 1 to 5) and a main session (digits from 1 to 9 ).

\section{Procedure}

The subjects first performed the interference control tasks. Afterwards, they performed the virtual scenarios with the order of presentation type (CAVE, display) counterbalanced across subjects. After completion of each virtual scenario, the subjects completed the presence inventory.

\section{Results}

\section{Presence scores}

The two virtual environments differed as to the intensity of subjective presence in the predicted way. Subjective presence was significantly higher in the high-immersion CAVE condition. This applied not only to the overall index of presence, but also to all components of subjective presence. Descriptive statistics on the subjective sense of presence and its components and the results of statistical analysis are presented in Table 1.

Table 1. Means (standard deviations) for the components of presence in different virtual environments and the results of statistical comparison.

\begin{tabular}{cccccc}
\hline & & \multicolumn{4}{c}{ Presence components } \\
& Presence & SP & N & EI & NE \\
\hline \multirow{2}{*}{ CAVE } & 128.6 & 2.93 & 3.35 & 2.73 & 2.15 \\
& $(17.9)$ & $(.47)$ & $(.61)$ & $(.63)$ & $(.72)$ \\
Display & 96.1 & 2.04 & 2.63 & 2.00 & 1.92 \\
& $(16.5)$ & $(.45)$ & $(.61)$ & $(.49)$ & $(.60)$ \\
Wilcoxon & -5.65 & -5.65 & -5.15 & -5.05 & -2.52 \\
T-test (Z) & $\mathrm{p}<0.001$ & $\mathrm{p}<0.001$ & $\mathrm{p}<0.001$ & $\mathrm{p}<0.001$ & $\mathrm{p}<0.05$ \\
\hline
\end{tabular}

\section{Flanker Task}

Non-parametric correlations between Flanker Task variables and components of presence are presented in Table 2. In the high-immersion CAVE condition, components of presence are related to average accuracy (overall presence, SP, EI, and NE components); accuracy in the incongruent condition (overall presence and NE component); and accuracy-related flanker interference cost (NE component). 
Other correlations were not significant even at a liberal $\mathrm{p}<0.1$ level. For the lowimmersion display conditions, almost all correlations were not significant. Only average accuracy in Flanker Task was related to the overall presence score. Generally, correlations between the efficiency of the Flanker Task and the components of presence were lower in the low-immersion display condition than in the CAVE condition.

Table 2. Non-parametric correlations between the efficiency of the Flanker Task and components of presence $\left({ }^{*}=\mathrm{p}<0.05,{ }^{\star}=\mathrm{p}<0.1\right)$.

\begin{tabular}{|c|c|c|c|c|c|}
\hline & \multicolumn{5}{|c|}{ Presence components } \\
\hline & Presence & SP & $\mathbf{N}$ & EI & NE \\
\hline & \multicolumn{5}{|c|}{ CAVE } \\
\hline $\mathrm{T}_{\mathrm{av}}$ & -.028 & -.047 & .091 & -.229 & .016 \\
\hline $\mathrm{A}_{\mathrm{av}}$ & $.519^{\star *}$ & $.455^{\star *}$ & .229 & $.421^{* *}$ & .256 \\
\hline $\mathrm{T}_{\text {con }}$ & .076 & -.106 & -.062 & -.253 & .016 \\
\hline $\mathrm{T}_{\mathrm{inc}}$ & -.004 & -.009 & .087 & -.240 & -.055 \\
\hline$A_{\text {con }}$ & -.231 & -.173 & -.207 & -.162 & .030 \\
\hline $\mathrm{A}_{\text {inc }}$ & $-.265^{\star}$ & -.258 & .004 & -.216 & $-.291^{*}$ \\
\hline $\mathrm{T}_{\mathrm{int}}$ & .106 & .148 & .035 & -.021 & .080 \\
\hline \multirow[t]{2}{*}{$\mathrm{E}_{\text {int }}$} & .213 & .215 & -.037 & .182 & $.281^{\star}$ \\
\hline & \multicolumn{5}{|c|}{ Display } \\
\hline $\mathrm{T}_{\mathrm{av}}$ & -.005 & .055 & -.121 & .127 & .105 \\
\hline $\mathrm{A}_{\mathrm{av}}$ & $.256^{\star}$ & -.144 & -.158 & -.108 & -.235 \\
\hline $\mathrm{T}_{\text {con }}$ & .002 & .069 & -.130 & .126 & .122 \\
\hline $\mathrm{T}_{\mathrm{inc}}$ & .043 & .053 & -.035 & .155 & .071 \\
\hline$A_{\text {con }}$ & -.176 & -.136 & -.124 & -.110 & -.028 \\
\hline $\mathrm{A}_{\text {inc }}$ & -.202 & -.149 & -.071 & -.108 & -.210 \\
\hline $\mathrm{T}_{\text {int }}$ & .072 & -.057 & -.130 & .007 & -.071 \\
\hline $\mathrm{E}_{\text {int }}$ & .168 & .123 & .045 & .082 & .144 \\
\hline
\end{tabular}

\section{Go/No Go task}

Non-parametric correlations between Go/No Go task variables and presence components are presented in Table 3. Under the high-immersion CAVE conditions, components of presence are related to average accuracy and the number of false alarms (EI component). In the low-immersion display condition, presence (SP component) is related to average reaction time. All other correlations were not significant even at a liberal $\mathrm{p}<0.1$ level, and generally correlations between Go-No Go task efficiency and components of presence were lower than the correlations between Flanker Task efficiency and components of presence. 
Table 3. Non-parametric correlations between the efficiency of Go/No Go task and components of presence $\left.{ }^{*}=\mathrm{p}<0.1\right)$

\begin{tabular}{cccccc}
\hline & \multicolumn{5}{c}{ Presence components } \\
\hline & Presence & SP & N & EI & NE \\
\hline $\mathrm{T}_{\mathrm{av}}$ & .009 & .173 & -.089 & -.133 & .039 \\
$\mathrm{~A}_{\mathrm{av}}$ & -.172 & -.100 & -.098 & $.311^{*}$ & -.140 \\
$\mathrm{~N}_{\mathrm{hit}}$ & -.025 & -.089 & .004 & -.098 & .058 \\
$\mathrm{~N}_{\mathrm{fa}}$ & .154 & .070 & .101 & $-.297^{*}$ & .139 \\
& & & CAVE & \\
$\mathrm{T}_{\mathrm{av}}$ & .138 & $-.293^{*}$ & -.033 & .077 & .055 \\
$\mathrm{~A}_{\mathrm{av}}$ & -.222 & -.132 & -.231 & -.213 & .115 \\
$\mathrm{~N}_{\mathrm{hit}}$ & -.048 & -.036 & .099 & -.093 & -.008 \\
$\mathrm{~N}_{\mathrm{fa}}$ & .199 & .100 & .226 & .192 & .126 \\
\hline
\end{tabular}

\section{Antisaccade task}

Non-parametric correlations between antisaccade task variables and components of presence are presented in Table 4. Under the high-immersion CAVE conditions the only correlation obtained was between SP component of presence and antisaccade task accuracy (this correlation was also increased in the display condition relative to most other correlations, but failed to reach the selected level of significance). Under the low-immersion display condition, there were no significant correlations between antisaccade task variables and components of presence. Overall, the correlations between the antisaccade task variables and components of presence were similar to that between the Go/No Go task variables and presence, and lower than that between the Flanker Task and presence.

Table 4. Non-parametric correlations between antisaccade task efficiency and components of presence $\left(^{*}=p<0.1\right)$.

\begin{tabular}{cccccc}
\hline & \multicolumn{5}{c}{ Presence components } \\
\hline & Presence & SP & N & EI & NE \\
\hline $\mathrm{T}_{\mathrm{av}}$ & -.010 & .012 & -.008 & -.127 & .205 \\
$\mathrm{~A}_{\mathrm{av}}$ & -.048 & $.261^{*}$ & .196 & -.164 & .030 \\
\hline & & & CAVE & \\
$\mathrm{T}_{\mathrm{av}}$ & & & Display & & .167 \\
$\mathrm{~A}_{\mathrm{av}}$ & -.006 & .074 & -.094 & .126 & -.113 \\
\hline
\end{tabular}




\section{Discussion}

In this study, the relationship between interference control and the subjective sense of presence while interacting with virtual environments was assessed in a sample of university students. The aim of the study was to check whether interference control-as a part of cognitive control-is a reliable determinant of presence. Several interference control tasks were used to measure the efficiency of various aspects of interference control: the Flanker Task, the Go/No Go task, and the antisaccade task. For instance, the Flanker Task addressed the efficiency of sensory and cognitive interference control, the Go/No Go task addressed the efficiency of cognitive and motor interference control, and the antisaccade task captured the efficiency of inhibiting inappropriate saccadic eye movements and control of overt attention. Besides investigation of the general relationship between interference control and presence, the study raised the interesting research question of whether different aspects of interference control are differentially related to various presence components.

We also contrasted two virtual environments. The CAVE environment is characterized by highly intense immersion of the participants and thus should produce conditions which promotes emergence of presence. The standard display environment is characterized by low immersion and thus is less able to promote a strong sense of presence. We checked whether interference control is differentially related to presence in virtual environments with different level of immersion. It is reasonable to assume that under different levels of immersion, there are different cognitive mechanisms which lead to interference control influencing presence.

The study results showed that performance in the Flanker Task is strongly related to various components of presence in the CAVE environment. Accuracy in the Flanker Task-reflecting the effectiveness of suppressing irrelevant visual stimuli competing for processing with the focal target stimuli-was strongly related to the overall index of presence, spatial presence component, and the emotional involvement component. That is, there seems to be a strong generalized relationship between presence and the effectiveness of irrelevant stimuli suppression in a highimmersion virtual environment. This result is in perfect accord with the notion that effectively directing a person's attention toward virtual environment objectively determines presence (Draper et al., 1998). The ability to voluntarily direct attention toward relevant stimuli is captured by the Flanker Task, and this individual ability may be the reason why different people experience different levels of presence in the same virtual environment. Sensory interference control is thus a possible determinant of presence, at least in highly immersive virtual environments.

The relationship between sensory interference control and presence is less articulated in low-immersion virtual environments. Here, a generalized relationship between the accuracy of the Flanker Task and the overall index of presence can be found, but it is significant only on a tendency level. While overall the results obtained support the idea that sensory interference control is a reliable determinant of presence, low-immersion virtual environments exhibit factors which attenuate this relationship. One factor is, obviously, the abundance of irrelevant visual stimuli in VR user's field of view which compete for processing resources much stronger than is the case in high-immersion virtual environments. This makes 
sensory interference control and covert attention control (both are accessed by the Flanker Task) less effective means of filtering out irrelevant stimulation. The latter operation is now more effectively accomplished by voluntarily restricting saccadic eye movements that results in a narrowing the efficient field of view. A second conclusion which may be drawn from the data is that the Flanker Task may reflect primarily sensory interference control though cognitive interference control would also be important for producing a strong feeling of presence. As there is much more potential for activating irrelevant representations (that is, representations irrelevant to the VR experience) in low-immersion environments than in high-immersion ones, sensory interference control becomes a less effective predictor; the relationship between effectiveness of the Flanker Task and presence is attenuated in this case.

The Go/No Go task also was related to presence, although to a lesser extent than the Flanker Task. The Go/No Go task reflects the ability to suppress responses that are usually permissible but become inappropriate in a given context. It was found that for the high-immersive CAVE environment, the accuracy of the Go/No Go task and the number of false alarms were related to the emotional involvement component of presence. For the low-immersion display condition, the Go/No Go task was unrelated to the emotional involvement but only to the spatial presence component. The fact that motor inhibition is generally related to presence, at least in high-immersion environments, seems justified. Control of an inappropriate response means the re-direction of attention toward the execution of appropriate response. Thus, effective motor interference control helps to shape overt actions with respect to the actual context, which produces a seamless interaction with the current (virtual) environment. This, in turn, reduces the emotional discomfort the user may experience when interacting with an unfamiliar virtual environment, which promotes the feeling of emotional involvement. If the ability to suppress inappropriate responses is limited, on the other hand, then interaction with the unfamiliar virtual environment is hindered, and there is emotional discomfort which disturbs the feeling of emotional involvement and presence generally. That this relationship does not hold for low-immersion environments again shows that presence is produced by different cognitive mechanisms in high-immersion and low-immersion environments. For instance, it may be that emotional involvement is generally weak in low-immersion environments, and floor effects hinder the creation of a relationship between motor interference control and emotional involvement.

In low-immersion environment, however, there is a link between Go/No Go task reaction time and spatial presence. The reaction time measure reflects the efficiency with which motor interference control demands are processed. That this general control efficiency measure is related to spatial presence may be explained by efficient motor interference control promoting a quick re-organization of spatial perception and action control in respect to the spatial specifics of a given virtual environment. Thus, the relationship we obtained may reflect a more general relationship between presence and cognitive control (see below).

Performance in the antisaccade task was only weakly related to presence. There was a tendency for statistical significance of the relationship between antisaccade task accuracy and spatial presence in the high-immersion environment, and no relationship between antisaccade task performance and presence in the low-im- 
mersion environment. Spatial presence refers to the aspects of presence related to the illusion of being transferred into another (virtual) space and acting there as if it were the real space. An obvious factor relating antisaccade task and spatial presence is that effective control of saccades restricts the user's efficient field of view to the virtual environment only. However, the cognitive mechanisms involved in the antisaccade task and presence may be more complex. Antisaccade task performance is considered to be an index of attention control (Munoz \& Everling, 2004) and the ability to maintain a goal in distractor-rich environments (Engle, 2002). Antisaccade task performance may thus be related to the general ability to perform goal-directed actions in an unfamiliar virtual environment in the presence of interference from the real environment. In this case, there is an overlap between mechanisms that produce the link between antisaccade task and presence, on the one hand, and between Flanker Task and presence, on the other. In this respect it is important to note that Flanker Task performance was also found to be related to spatial presence.

In general, our data suggest that interference control generally is related to presence, although this link is mostly pronounced for sensory interference control and for high-immersion virtual environments. These findings help explain why constructs only distantly related to presence like working memory capacity (WMC) have been shown to correlate with presence (Rawlinson et al., 2012). WMC has repeatedly been shown to be related to the efficiency of interference control (Engle, 2002), and the link between WMC and presence may be due to the involvement of interference control in both cognitive processes. As WMC is especially related to control of cognitive interference, this aspect of interference control-only partially represented in the present study-may be another important determinant of presence. It is a task for future research to show this link by unequivocally operationalizing cognitive interference control (for example, by the means of proactive interference control memory tasks).

The relationship we found between interference control and presence may also indicate that there is a connection between cognitive control (of which interference control is an important aspect) and the subjective feeling of presence in virtual environments. Cognitive control is conceptualized as the group of brain processes responsible for context-dependent cognitive system re-configuration toward the task at hand. As such, cognitive control is excellently suited for the task of tuning the cognitive system toward interaction with a virtual environment substituted for the real environment. Our study demonstrated how this theoretical conclusion can be experimentally elaborated by finding links between presence and diverse aspects of cognitive control. It is necessary to note that neurocognitive research also support the notion that there is a link between cognitive control and presence. For instance, Jäncke et al. (2009) have shown that prefrontal cortex activity associated with cognitive control may be associated with subjective feeling of presence.

\section{Conclusion}

In this study, the relationship between interference control and aspects of presence was investigated. It was found that interference control is generally related to the sense of presence. This link was especially strong for high-immersion virtual en- 
vironments. From the various aspects of interference control, sensory interference control was most strongly associated with various aspects of presence (overall presence score, spatial presence, and emotional involvement). Sensory interference control exerts its influence on presence by allowing the user to concentrate on stimuli pertaining to the virtual environment, and to ignore irrelevant stimuli pertaining to the real environment. Motor interference control was associated with spatial presence and emotional involvement, but this relationship was less strong than was the case with sensory interference control. Motor interference control exerts its influence on presence by allowing the user to concentrate on VR appropriate actions, thus making the VR-user interaction more natural. Low-immersion virtual environments attenuate some of these links between interference control and presence so that only sensory interference control remains a notable predictor of presence in this case. The relationship we found between interference control and presence may reflect a more general relationship between cognitive control and presence.

\section{Acknowledgments}

This study is supported by the Russian Foundation for Basic Research, RFFR grant 15-06-08998.

\section{References}

Baños, R., Botella, C., Garcia-Palacios, A., Villa, H., Perpina, C., \& Gallardo, M. (1999). Psychological variables and reality judgements in virtual environments: The role of absorption and dissociation. Cyberpsychology and Behavior, 2, 135-142. doi: 10.1518/001872098779591386

Draper, J., Kaber, D., \& Usher, J. (1998). Telepresence. Human Factors, 40, 354-375. doi: $10.1518 / 001872098779591386$

Diemer, J., Alpers, G., Peperkorn, H., Shiban, Y., \& Mühlberger, A. (2015). The impact of perception and presence on emotional reactions: A review of research in virtual reality. Frontiers in Psychology, 6, 26. doi: 10.3389/fpsyg.2015.00026

Engle, R. (2002). Working memory capacity as executive attention. Current Directions in Psychological Science, 11, 19-23. 10.1111/1467-8721.00160

Friedman, N., \& Miyake, A. (2004). The relations among inhibition and interference control functions: a latent-variable analysis. Journal of Experimental Psychology: General, 133, 101135. doi: 10.1037/0096-3445.133.1.101

Hasher, L., \& Zacks, R. (1988). Working memory, comprehension, and aging: A review and a new view. In G.H. Bower (Ed.), The Psychology of Learning and Motivation, Vol. 22 (pp. 193225). New York: Academic Press. doi: 10.1016/S0079-7421(08)60041-9

Jäncke, L., Cheetham, M., \& Baumgartner, T. (2009). Virtual reality and the role of the prefrontal cortex in adults and children. Frontiers in Neuroscience, 3, 52-59. doi: 10.3389/ neuro.01.006.2009

Lessiter, J., Freeman, J., Keogh, E., \& Davidoff, J. (2001). A cross-media presence questionnaire: The ITC-Sense of Presence Inventory. Presence: Teleoperators and Virtual Environments, 10, 282-297. doi: 10.1162/105474601300343612

Lombard, M., \& Ditton, T. (1997). At the heart of it all: The concept of presence. Journal of Computer Mediated Communication, 3. Retrieved from http://onlinelibrary.wiley.com/ doi/10.1111/j.1083-6101.1997.tb00072.x/abstract 10.1111/j.1083-6101.1997.tb00072.x 
Miyake, A., Friedman, N., Emerson, M., Witzki, A., Howerter, A., \& Wager, T. (2000). The unity and diversity of executive functions and their contributions to complex "Frontal Lobe" tasks: A latent variable analysis. Cognitive Psychology, 41, 49-100. doi: 10.1006/cogp.1999.0734

Munoz, D., \& Everling, S. (2004). Look away: The anti-saccade task and the voluntary control of eye movement. Nature Reviews Neuroscience, 5, 218-228. doi: 10.1038/nrn1345

Notebaert, W, \& Verguts, T. (2008). Cognitive control acts locally. Cognition, 106, 1071-1080. doi: 10.1016/j.cognition.2007.04.011

Rawlinson, T., Lu, Sh., \& Coleman, P. (2012). Individual differences in working memory capacity and presence in virtual environments. In H. Zhang, A. Hussain, D. Liu \& Zhanshan Wang (Eds.), Advances in brain inspired cognitive systems. Lecture Notes in Computer Science, vol. 7366, (pp. 22-30). Heidelberg/New York: Springer. doi: 10.1007/978-3-642-31561-9_3

Sacau, A., Laarni, J., \& Hartmann, T. (2008). Influence of individual factors on presence. Computers in Human Behavior, 24, 2255-2273. doi: 10.1016/j.chb.2007.11.001

Sanchez-Vives, M., \& Slater, M. (2005). From presence to consciousness through virtual reality. Nature Reviews Neuroscience, 6, 332-339. doi: 10.1038/nrn1651

Sas, C. (2004). Individual differences in virtual environments. Computational science - ICCS 2004, Fourth international conference, proceedings, part III. Lecture Notes in Computer Science, vol. 3038, (pp. 1017-1024). Heidelberg/New York: Springer. doi: 10.1007/978-3-54024688-6_131

Velichkovsky, B.B. (2014). Psychological factors of the emerging sense of presence in virtual environments. National Psychological Journal, 3, 31-38. doi: 10.11621/npj.2014.0304

Original manuscript received August 22, 2016

Revised manuscript accepted January 25, 2017

First published online September 30, 2017 